\title{
Circulating-free tumour DNA and the promise of disease phenotyping in hepatocellular carcinoma
}

\author{
David J. Pinato $\mathbb{1}^{1}$
}

Received: 17 March 2018 / Accepted: 23 March 2018 / Published online: 14 May 2018

(c) Macmillan Publishers Limited, part of Springer Nature 2018

The management of hepatocellular carcinoma (HCC), the third most lethal solid tumour, is currently limited by the incomplete dissection of the pharmacologically actionable drivers underlying its clinical progression. In stark contrast to lung, colorectal cancer, melanoma and many other malignancies where molecularly based disease stratification has led to improvements in survival through a perfected allocation of available treatments, HCC has remained relatively untouched by the 'molecular revolution' that has affected cancer therapeutics over the past two decades [1]. Whilst HCC is acknowledged as a molecularly composite disease, previously published tissue genomic signatures have been largely descriptive and substantially unhelpful to qualify stratifying biomarkers to optimise clinical decision making [2]. Whole-exome sequencing studies have confirmed an overall rate of two mutations per megabase in HCC, with a median of 45 non-synonymous variants, mostly affecting genes related to DNA repair, cell cycle progression and chromatin remodelling including TP53, CTNNB1 and many others [3]. Evidence of potentially 'actionable' mutations is present in up to $28 \%$ of tumours, with little evidence, though, regarding their true oncogenic potential [4].

In this issue of Oncogene, Lagbaa et al. [5] present the results of a pilot study of ultra-deep sequencing of circulating-free tumour DNA (ctDNA) in a small cohort of early-stage HCC patients. Using a targeted exome sequencing approach followed by orthogonal validation by digital droplet polymerase chain reaction (PCR), the authors confirm serum and plasma detection of somatic variants spanning from renowned HCC-specific pathogenic loci such as TERT promoter, TP53 and CTNNB1, as well as a number

David J. Pinato

david.pinato@imperial.ac.uk

1 Department of Surgery and Cancer, Imperial College London, Hammersmith Hospital, Du Cane Road, London W12 0NN, UK of oncogenes for which targeted therapies exist including JAK1, PDGFRB and BRAF. In addition, the authors demonstrate the high concordance between ctDNA and tissue-based profiling, qualifying ctDNA as surrogate biomarker of tumour mutational status.

Compared to other malignancies, the prospect of a 'liquid biopsy' is of greatest appeal in HCC, a disease where histologic confirmation of the diagnosis is rarely sought [6], depriving clinicians of an opportunity to incorporate the molecular makeup of the tumour in clinical decision making.

By re-conducting the role of ctDNA to the Barcelona Clinic Liver Cancer (BCLC) model, the staging algorithm that guides prognostication and treatment allocation in HCC (Fig. 1), ctDNA shows great potential in dissecting the clinical heterogeneity that characterises patients with HCC across a broad range radical and palliative treatments [6].

Following radical therapies such as tumour resection, ablation or liver transplantation, longitudinal monitoring of HCC-specific somatic variants is expected to improve risk stratification by enabling pre-symptomatic identification of relapse. Whilst the high reproducibility observed between ctDNA and tumour genomic features described in this study is certainly promising, ctDNA-based identification of minimal residual disease (MRD) in HCC might be biologically and conceptually different from leukaemias and lymphomas, a setting where the level of MRD is an established guide to prognosis and relapse risk [7]. First, markers used for DNA-based tests of MRD are often chromosomal translocations that are highly oncogenic, universally present and easily detectable with PCR-based techniques [8]. In contrast, the pathogenesis of HCC cannot be traced to a single, specific genetic abnormality [9]. Whilst panels of HCC-specific point mutations have been optimised [10], the reliance on next-generation sequencing for their identification might represent a technical and a cost barrier to the routine implementation of ctDNA-based diagnostics, more so if ultra-deep, high-coverage approaches are required. 


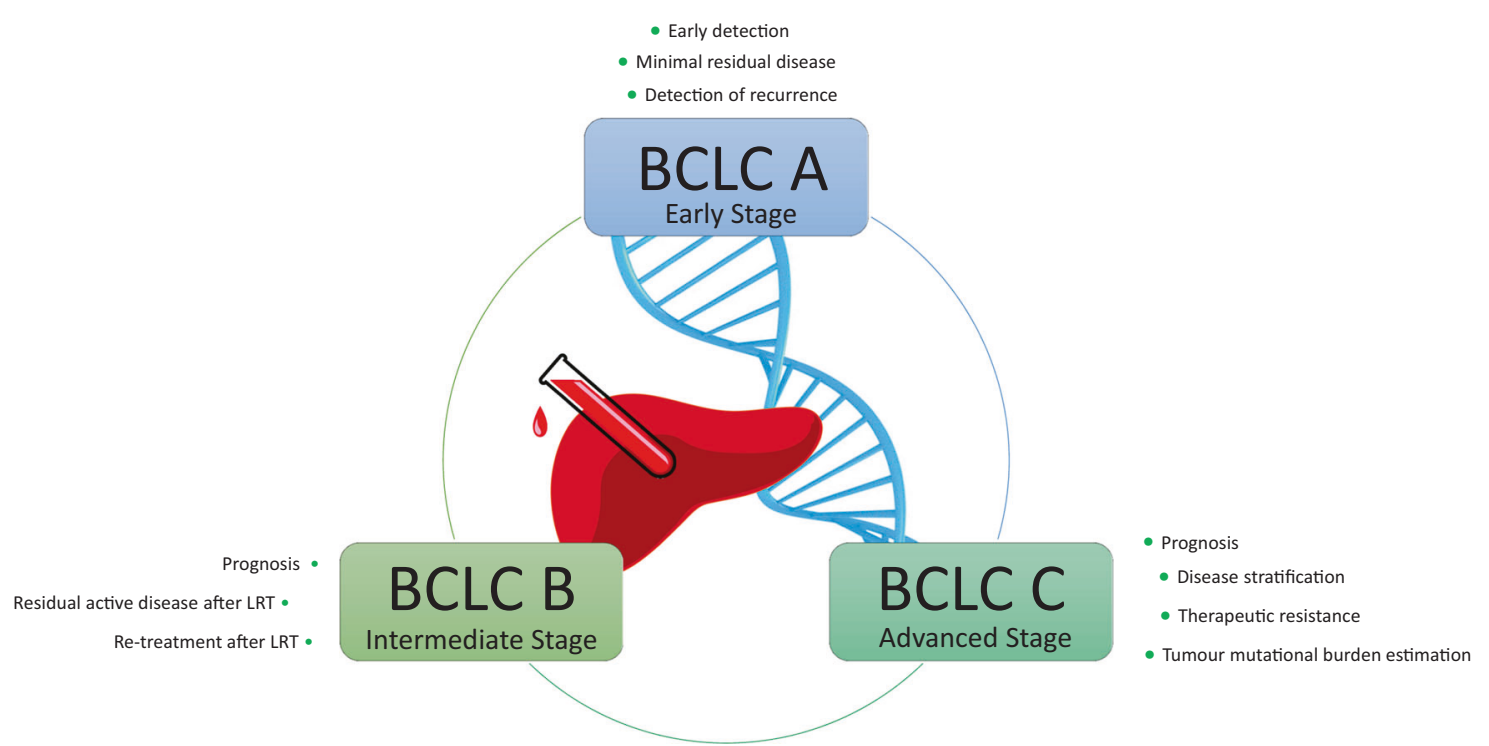

Fig. 1 Schematic representation of the potential applications of ctDNA-based diagnostics across the various Barcelona Clinic Liver Cancer stages of HCC. LRT loco-regional therapies

A second and perhaps even more crucial issue that might affect surrogacy of ctDNA in portraying the genomic features of HCC is intra-tumour heterogeneity (ITH) [11]. ITH is a recognised feature of evolving malignancies where the diverse coexisting neoplastic sub-clones accumulate serial genetic and epigenetic modifications in space and time, with ultimate implications in their differential sensitivity to treatment [12] and impact on patients survival [13]. Evolving evidence in early-stage HCC has unveiled a significant degree of ITH in $87 \%$ of HCCs, as defined by immunehistological criteria and mutational status of highly prevalent mutational drivers such as TP53 and CTNNB1 [14].

Whilst limited by the choice of a small cohort of earlystage patients, where ITH might be a less prominent feature compared to advanced disease, an important contribution of the study by Lagbaa is the confirmation of a clonal distribution of TP53, CTNNB1 and TERT promoter mutations using multi-region sequencing, mirrored by evidence of sub-clonal variants in a subset of loci including APOB, ALB and BRAF.

The impact of clonality is of greater consequence in the qualification of ctDNA as a clinically available test for the diagnosis and therapeutic monitoring of HCC, more so in light of the multi-factorial nature of HCC relapse. Following resection or tumour ablation, early intra-hepatic recurrence is traditionally attributed to true disease re-occurrence after radical treatment as opposed to de novo tumour formation [15]. Whether ctDNA might capture the genomic heterogeneity between primary, relapsed disease or de novo disease re-occurrence remains to be tested in prospective studies and its performance in this setting is likely to dictate the fate of ctDNA as a biomarker in early-stage HCC.
In this context, the yet unaddressed question of tumourspecific origin of mutations identified in circulating-free DNA (cfDNA) adds a further layer of complexity to the biologic qualification of this biomarker. With systemic DNA release being directly proportional to tissue turnover and necro-inflammatory injury, the relative contribution of the pro-inflammatory cirrhotic milieu in determining qualitative and quantitative changes in cfDNA deserves further investigation [16]. A number of studies have confirmed that cfDNA can be detected in a number of non-malignant conditions including, for instance, non-alcoholic fatty liver disease [17]. This reinforces the need for comprehensive genomic profiling of patients with cirrhosis, where detection of somatic variants in circulating DNA released from preneoplastic hepatocytes might be a reflection of the 'field cancerisation' process typical of the progression from endstage liver disease to HCC [18].

The potential benefits of ctDNA in the HCC clinic are reaching far beyond early diagnosis and therapeutic monitoring after curative treatment. In intermediate-stage HCC (BCLC B), the detection of residual active disease following loco-regional therapy with trans-arterial chemoembolisation (TACE) can be challenging. A subset of BCLC B patients, in fact, do not respond to TACE or become refractory after initial treatment and despite attempts to identify non-responders using clinical or imaging parameters, characterisation of this poor prognostic group remains elusive. Multiple levels of evidence suggest ctDNA to reflect tumour burden [10] and dynamic changes in the allelic frequency of HCC-specific mutations could serve as a minimally invasive test integrating with imaging reassessment to guide the need for further re-treatment. 
Perhaps, a far more appealing translational implication that develops from this preliminary study is the promise of ctDNA to act a source of disease-stratifying genomic biomarkers for systemic therapy. A decade after the approval of sorafenib as the first systemic agent capable of producing a significant survival benefit [19], the provision of systemic anticancer treatment in HCC is still unsupported by predictive correlates of response [20], a point of greater consequence now that treatment options for HCC are expanding [21]. The lack of molecularly defined disease phenotyping has adversely impacted drug development, which has recognised a period of profound stagnation in the last decade [22].

CtDNA-based disease phenotyping has rapidly reached the clinic in non-small cell lung cancer, where identification of epidermal growth factor receptor (EGFR) T790M mutations in peripheral blood has become an Food and Drug Administration-approved test to facilitate insight into therapeutic resistance to first-generation EGFR inhibitors [23].

Whilst similar candidate-based approaches using BEAMing technology have been reproduced in malignancies with robust genomic classifiers such as RAS/RAF/ PI-3-kinase mutant colorectal cancer [24], the genomic complexity of HCC makes plasma genotyping far more challenging. In phase II trials of refametinib in RAS mutant HCC, for instance, ctDNA-based screening of RAS mutations demonstrated to be a feasible strategy to enrich for molecularly defined patient populations. However, the low prevalence of RAS mutations $(\sim 6 \%)$ required screening of $>1300$ plasma samples to derive a small cohort of 16 RAS mutant patients [25], highlighting the challenge of adopting somatic variants as response predictors in a disease where the majority of core 'actionable' mutations have a prevalence of $<10 \%$ and a fairly undefined linkage with response to targeted inhibition [26]. The clinical development of programmed cell-death 1-targeted immune checkpoint inhibitors, for which no reliable response predictor exists in HCC, might overcome the issues surrounding the low prevalence of 'actionable' variants and high prevalence of 'undruggable' mutations, by affording ctDNA the role of minimally invasive and dynamic estimate of the overall tumour mutational burden, a renown determinant of response to immunotherapy [27].

As ctDNA becomes a more prominent source of genomic information across malignancies, the study by Lagbaa published in this issue of Oncogene represents a preliminary, yet important step in the biomarker qualification roadmap for potential diagnostic, prognostic and predictive applications of ctDNA in HCC. Prospective studies should validate whether ctDNA will hold the promise of improving disease phenotyping and address the uncertainties imposed by a rapidly evolving treatment landscape in HCC by deciphering its heterogeneous molecular pathophysiology.

Acknowledgements DJP is supported by grant funding from the National Institute for Health Research (NIHR), the Academy of Medical Sciences (AMS) and Cancer Research UK (CRUK).

\section{Compliance with ethical standards}

Conflict of interest The authors declare that they have no conflict of interest.

\section{References}

1. Nault JC, Galle PR, Marquardt JU. The role of molecular enrichment on futuretherapies in hepatocellular carcinoma. J Hepatol. 2018; pii: S0168-8278(18)30134-X; epub ahead of print 2 March 2018; https://doi.org/10.1016/j.jhep.2018.02.016.

2. Hoshida Y, Toffanin S, Lachenmayer A, Villanueva A, Minguez B, Llovet JM. Molecular classification and novel targets in hepatocellular carcinoma: recent advancements. Semin liver Dis. 2010;30:35-51.

3. Cleary SP, Jeck WR, Zhao X, Chen K, Selitsky SR, Savich GL, et al. Identification of driver genes in hepatocellular carcinoma by exome sequencing. Hepatology. 2013;58:1693-702.

4. Schulze K, Imbeaud S, Letouze E, Alexandrov LB, Calderaro J, Rebouissou S, et al. Exome sequencing of hepatocellular carcinomas identifies new mutational signatures and potential therapeutic targets. Nat Genet. 2015;47:505-11.

5. Labgaa I, Villacorta-Martin C, D'Avola D, Craig AJ, von Felden J, Martins-Filho SN, et al. A pilot study of ultra-deep targeted sequencing of plasma DNA identifies driver mutations inhepatocellular carcinoma. Oncogene; epub ahead of print 9 April 2018; https://doi.org/10.1038/s41388-018-0206-3.

6. Bruix J, Reig M, Sherman M. Evidence-based diagnosis, staging, and treatment of patients with hepatocellular carcinoma. Gastroenterology. 2016;150:835-53.

7. Buccisano F, Maurillo L, Del Principe MI, Di Veroli A, De Bellis E, Biagi A, et al. Minimal residual disease as a biomarker for outcome prediction andtherapy optimization in acute myeloid leukemia. Expert Rev Hematol. 2018;11:307-13.

8. Yeung CC, Egan D, Radich JP. Molecular monitoring of chronic myeloid leukemia: present and future. Expert Rev Mol Diagn. 2016;16:1083-91.

9. Kancherla V, Abdullazade S, Matter MS, Lanzafame M, Quagliata L, Roma G, et al. Genomic analysis revealed new oncogenic signatures in TP53-mutant hepatocellular carcinoma. Front Genet. 2018;9:2

10. Ikeda S, Lim JS, Kurzrock R. Analysis of tissue and circulating tumor DNA by next generation sequencing of hepatocellular carcinoma: implications for targeted therapeutics. Mol Cancer Ther; epub ahead of print 26 February 2018; https://doi.org/10. 1158/1535-7163.MCT-17-0604.

11. Zhai W, Lim TK, Zhang T, Phang ST, Tiang Z, Guan P, et al. The spatial organization of intra-tumour heterogeneity and evolutionary trajectories of metastases in hepatocellular carcinoma. Nat Commun. 2017;8:4565.

12. Swanton C. Intratumor heterogeneity: evolution through space and time. Cancer Res. 2012;72:4875-82.

13. Burrell RA, Swanton C. Tumour heterogeneity and the evolution of polyclonal drug resistance. Mol Oncol. 2014;8:1095-111. 
14. Friemel J, Rechsteiner M, Frick L, Bohm F, Struckmann K, Egger $\mathrm{M}$, et al. Intratumor heterogeneity in hepatocellular carcinoma. Clin Cancer Res. 2015;21:1951-61.

15. Sherman M. Recurrence of hepatocellular carcinoma. N Engl J Med. 2008;359:2045-7.

16. Howell JA, Khan SA, Knapp S, Thursz MR, Sharma R. The clinical role of circulating free tumor DNA in gastrointestinal malignancy. Transl Res. 2017;183:137-54.

17. Karlas T, Weise L, Kuhn S, Krenzien F, Mehdorn M, Petroff D, et al. Correlation of cell-free DNA plasma concentration with severity of non-alcoholic fatty liver disease. J Transl Med. 2017;15:106.

18. Piciocchi M, Cardin R, Vitale A, Vanin V, Giacomin A, Pozzan $\mathrm{C}$, et al. Circulating free DNA in the progression of liver damage to hepatocellular carcinoma. Hepatol Int. 2013;7:1050-7.

19. Llovet JM, Ricci S, Mazzaferro V, Hilgard P, Gane E, Blanc JF, et al. Sorafenib in advanced hepatocellular carcinoma. N Engl J Med. 2008;359:378-90.

20. Pinato DJ, Breaking Kuhn's paradigm in advanced hepatocellular carcinoma. Hepatology. 2018;67:1663-5.

21. Gerbes A, Zoulim F, Tilg H, Dufour JF, Bruix J, Paradis V, et al. Gut roundtable meeting paper: selected recent advances in hepatocellular carcinoma. Gut. 2018;67:380-8.
22. Maida M, Iavarone M, Raineri M, Camma C, Cabibbo G. Second line systemic therapies for hepatocellular carcinoma: reasons for the failure. World J Hepatol. 2015;7:2053-7.

23. Oxnard GR, Thress KS, Alden RS, Lawrance R, Paweletz CP, Cantarini $\mathrm{M}$, et al. Association between plasma genotyping and outcomes of treatment with osimertinib (AZD9291) in advanced non-small-cell lung cancer. J Clin Oncol. 2016;34:3375-82.

24. Tabernero J, Lenz HJ, Siena S, Sobrero A, Falcone A, Ychou M, et al. Analysis of circulating DNA and protein biomarkers to predict the clinical activity of regorafenib and assess prognosis in patients with metastatic colorectal cancer: a retrospective, exploratory analysis of the CORRECT trial. Lancet Oncol. 2015;16:937-48.

25. Llovet JM. Phase II studies with refametinib or refametinib plus sorafenib in patients with mutant RAS hepatocellular carcinoma (HCC). Hepatology. 2016;64:601-810.

26. Shibata T, Aburatani H. Exploration of liver cancer genomes. Nat Rev Gastroenterol Hepatol. 2014;11:340-9.

27. Khagi Y, Goodman AM, Daniels GA, Patel SP, Sacco AG, Randall JM, et al. Hypermutated circulating tumor DNA: correlation with response to checkpoint inhibitor-based immunotherapy. Clin Cancer Res. 2017;23:5729-36. 